\title{
First-visit patients without a referral to the Department of Internal Medicine at a medium-sized acute care hospital in Japan: an observational study
}

This article was published in the following Dove Press journal:

International Journal of General Medicine

4 October 2017

Number of times this article has been viewed

\author{
Nobuyuki Kajiwara' \\ Kazuyuki Hayashi' \\ Masahiro Misago ${ }^{2}$ \\ Shinichiro Murakami ${ }^{2}$ \\ Takato Ueoka ${ }^{2}$ \\ 'Department of Nephrology, Ikeda \\ City Hospital, ${ }^{2}$ Department of \\ General Medicine, Ikeda City Hospital, \\ Johnan, Osaka, Japan
}

Purpose: We sought to profile first-time patients without a referral who sought medical care at the Department of Internal Medicine at a medium-sized acute care hospital in Japan. We anticipated that the analysis would highlight the demand for medical care needs from acute care hospitals and help confirm one of the problems associated with primary care in Japan.

Patients and methods: The study population comprised 765 patients who sought outpatient consultation without a referral at "the Department of General Internal Medicine" at the Ikeda City Hospital on Fridays over 4 years. Data on the following variables were collected: age, sex, examination date, reason for encounter (RFE), diagnosis, as well as history of consultation with or without antibiotic treatment at another medical institution for the same RFE. We used the International Classicication of Primary Care, Revised Second edition (ICPC-2-R) codes for RFEs and diagnoses.

Results: The main RFE fields were digestive (ICPC-2-R Chapter D), general and unspecified $(A)$, and respiratory $(\mathrm{R})$. The main diagnosis fields were digestive (D), respiratory $(\mathrm{R})$, general and unspecified (A), and musculoskeletal (L). In total, $27.6 \%$ of patients had sought consultation at another medical institution for the same RFE. Of these, $64.7 \%$ of patients for whom the RFE was cough (ICPC-2-R code, R05), and $72.0 \%$ for whom the RFE was fever (A03) were prescribed antibiotics. In total, $62.4 \%$ of patients underwent emergency investigations and waited for the results; $4.3 \%$ were hospitalized on the same day; and $60.5 \%$ were medicated at the initial examination. In $11.5 \%$, the main underlying problem appeared to be psychosomatic. Conclusion: We used the ICPC-2-R to analyze the state of first-visit patients without a referral visiting the Department of Internal Medicine at a medium-sized acute care hospital in Japan. Common RFEs were abdominal pain, cough, and fever. A tendency toward overprescription of antibiotics was observed among primary care physicians.

Keywords: medium-sized hospital, general internal medicine, general practice, reason for encounter, International Classification of Primary Care, antibiotic overuse

\section{Introduction}

In Japan, the national health insurance system ensures free access to almost all medical institutions. ${ }^{1,2}$ There is no clear boundary between primary and secondary care, and no one acts as a gatekeeper between them. ${ }^{3}$ However, due to the escalating medical costs and an increase in the aging population in Japan, most of the acute care hospitals are focusing on providing the emergency and inpatient treatment services rather than outpatient services. Ideally, only patients who come with a referral from a primary care clinic should be accepted as outpatients in such hospitals.
Correspondence: Nobuyuki Kajiwara Department of Nephrology, Ikeda City Hospital, 3-I-18 Johnan, Ikeda, Osaka 563-85I0, Japan

$\mathrm{Tel}+8|7275| 288 \mid$

Fax +81727546380

Email nkajiwara@ams.odn.ne.jp 
A primary care physician writes a referral to an acute care hospital when he or she determines that a more specialized level of care be necessary. However, in the current system, some patients seek consultation at acute care hospitals on their own without a referral. Acute care hospitals that have fulfilled certain requirements are allowed to collect a "fee for treatment of patients' choice" from first-visit patients without a referral. ${ }^{4}$

Ikeda City Hospital is 18 min by train from Osaka city the second largest city in Japan. It is a 364-bedded hospital, providing acute care services. Ikeda city has a population of 100,000. Ikeda City Hospital is the biggest hospital in Ikeda city. First-time walk-in patients without referral letters are required to pay a fee of $¥ 2160$ for "treatment of patients' choice" in addition to their national health insurance co-payment. Referral rates are used for calculation of medical fees at hospitals. The mean annual referral rate for all first-visit patients to the hospital was $66.8 \%$ (referral rate from April 2012 to March 2014=[number of referred patients + number of same-day hospitalization first-visit patients]/[number of first-visit patients - number of holiday and nighttime first-visit patients + number of holiday and nighttime same-day hospitalization first-visit patients]; referral rate from April 2014 onward = number of referred patients/[number of first-visit patients - number of patients transported to hospital by ambulance - number of holiday and nighttime first-visit patients - number of first-visit patients with no subjective symptoms whose treatment was started after a disease was detected at an examination for a health checkup]) between April 2012 and March 2016. The Japanese government changed the calculation method in April 2014. Between those years, the annual referral rates to our hospital were $64.2 \%, 62.4 \%, 67.2 \%$, and $73.2 \%$, respectively.

As a rule, the treatment of patients with internal medicine diagnoses who underwent an examination without a referral at the Ikeda City Hospital was overseen by "the Department of General Internal Medicine." These patients paid the "fee for treatment of patients' choice" to the Ikeda City Hospital as well as first-visit fees to another medical institution if they visited one.

The main objective of this study was to gain insight into medical demands and patient characteristics of those who came in as walk-in patients to "the Department of General Internal Medicine" at the Ikeda City Hospital without a referral from a primary care clinic. Another objective of this study was to highlight the problem of overprescription of antibiotics by primary care providers in Japan.

\section{Patients and methods}

\section{Patients}

This study included all patients who presented for the first time to our medical outpatient clinic without a referral letter, on Fridays between April 2012 and March 2016, to include 765 patients who were randomly assigned to 1 of 2 examination rooms in order of arrival and underwent an examination by the author (NK). We excluded patients who were transported to the hospital by ambulance, were $<16$ years old, or were patients who sought examinations from the Departments of Surgery; Obstetrics and Gynecology; Orthopedics; Dermatology; and Eye, Ear, Nose, Throat, or Dental Surgery. We included patients who received a simple written recommendation to undergo an examination with an explanation of their results from a health checkup, but we did not include patients who were provided with an actual referral letter from a doctor.

\section{Ethics approval}

This study was approved by the institutional review board of the Ikeda City Hospital. The approval number is A28004. The data accessed in the medical records were de-identified in this manuscript. Because this manuscript contains no individual person's data, and this study is observational and noninterventional, the institutional review board of the Ikeda City Hospital waived patient consent.

\section{Measurements}

We investigated age; sex; examination date; reason for encounter (RFE); International Classification of Primary Care, Revised Second edition (ICPC-2-R) codes for RFE (mainly, "Component 1 - Complaint and symptom component"); ${ }^{5}$ and diagnosis; ICPC-2-R codes for diagnosis (mainly, "Component 7- Diagnosis/disease component"); whether the patients had been examined at another medical institution for the same RFE; whether they had been administered antibiotics when examined for the same RFE at another medical institution; whether they were examined during a health checkup; whether they underwent emergency testing on the initial examination day; the outcome of the initial examination; whether our hospital administered any medication on the initial examination day; and whether the patients appeared eligible for psychosomatic treatment. For simplification, we selected the one most important complaint of each patient as the RFE. Then, we reviewed the patients' medical records for the subsequent 2 months to confirm that the 1 diagnosis correlated with the RFE. Plain radiography, 
blood testing, or other tests were considered to be emergency testing when treatment on that day was not concluded until the results were received. The patients' eligibility for psychosomatic treatment was determined on the basis of the recorded subjective impression of the doctor conducting the examination. No specific scores or criteria for the diagnosis of psychosomatic diseases were used.

\section{Data analysis}

Ages are presented as mean \pm SD. Other data are only presented as the percentages of the study population. No statistical test was used.

\section{Results}

The mean age of the 765 patients was $49.4 \pm 18.7$ years. There were 349 male ( $49.6 \pm 18.5$ years) and 416 female patients (49.1 \pm 18.9 years). Sixteen patients were examined twice on separate days for different RFEs. In these cases, the patients were counted for each examination.

As shown in Figure 1, the most common RFE by field (corresponding to chapters in ICPC-2-R) was digestive (D) (hereinafter, single alphabet letters are used to represent corresponding ICPC-2-R chapters), followed by general and unspecified (A), respiratory (R), and neurological $(\mathrm{N})$. The top 30 RFEs are shown in Table 1. These included, in order beginning with the most common, cough (R05) (hereinafter, combinations of a single alphabet letter and 2 Arabic numerals signify ICPC-2-R codes); fever (A03); abdominal pain, epigastric (D02); and abdominal pain, localized, other (D06). However, when abdominal pain, epigastric (D02); abdominal pain, localized, other (D06); and abdominal pain/cramps, general (D01) were combined into a total abdominal pain category, this became the most common RFE (105 patients; $13.7 \%)$.

A breakdown of the diagnoses by field (chapter) is shown in Figure 2. The most common was digestive (D), followed by respiratory (R), general and unspecified (A), and musculoskeletal (L). Table 2 lists the top 50 diagnoses. Common diagnoses included upper respiratory infection, acute (R74); gastroenteritis, presumed infection (D73); stomach function disorder (D87); and influenza (R80). Gastroenteritis, presumed infection (D73) included so-called acute gastroenteritis and acute enteritis. Stomach function disorder (D87) included patients who exhibited chronic gastritis on upper gastrointestinal endoscopy, and esophagus disease (D84) included reflux esophagitis. Of the 765 patients, the RFE code was the same as the diagnosis code in 211 patients (27.6\%). The most common codes for these 211 patients were headache (N01, n=14); abdominal pain, localized, other (D06, $\mathrm{n}=13$ ); cough ( $\mathrm{R} 05, \mathrm{n}=12)$; abdominal pain, epigastric (D02,

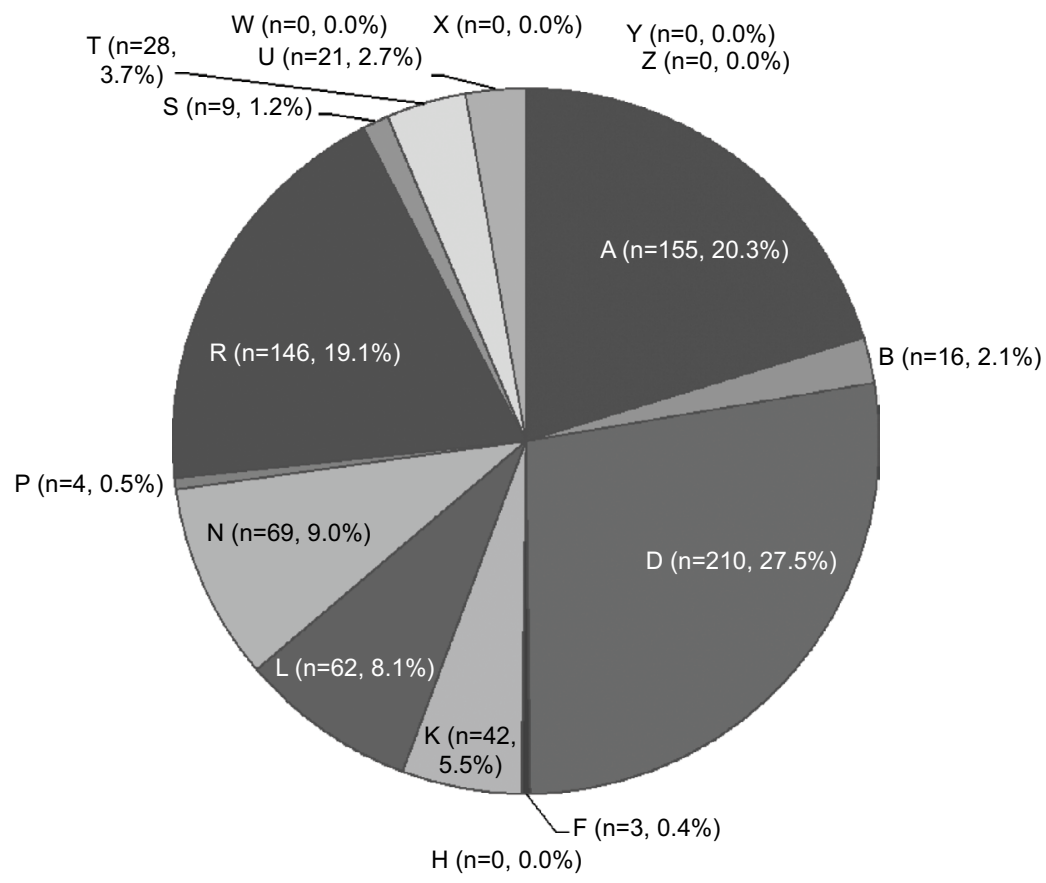

Figure I Frequency of RFEs classified by the ICPC-2-R for 765 first-visit patients who presented to the Department of Internal Medicine at the Ikeda City Hospital without a referral on Fridays between April 2012 and March 2016.

Notes: $A=$ general and unspecified; $B=$ blood, blood-forming organs, and immune mechanisms; $D=$ digestive; $F=$ eye; $H=$ ear; $K=$ circulatory; $L=$ musculoskeletal; $N=$ neurological; $\mathrm{P}=$ psychological; $\mathrm{R}=$ respiratory; $\mathrm{S}=$ skin; $\mathrm{T}=$ endocrine, metabolic, and nutritional; $\mathrm{U}=$ urological; $\mathrm{W}=$ pregnancy, child-bearing, and family planning; $\mathrm{X}=$ female genital; $Y=$ male genital; $Z$ = social problems.

Abbreviations: ICPC-2-R, International Classification of Primary Care, Revised Second edition; RFE, reason for encounter. 
Table I Top 30 ICPC-2-R RFE codes of first-visit patients who presented to the Department of Internal Medicine at the Ikeda City Hospital without a referral on Fridays between April 2012 and March 2016

\begin{tabular}{|c|c|c|c|c|c|}
\hline Ranking & $\begin{array}{l}\text { ICPC-2-R } \\
\text { code }\end{array}$ & ICPC-2-R title & $\begin{array}{l}\text { Number of } \\
\text { cases }\end{array}$ & Percentage & $\begin{array}{l}\text { Cumulative } \\
\text { percentage }\end{array}$ \\
\hline $\mathrm{I}$ & R05 & Cough & 88 & 11.5 & 11.5 \\
\hline 2 & $\mathrm{~A} 03$ & Fever & 85 & 11.1 & 22.6 \\
\hline 3 & D02 & Abdominal pain, epigastric & 55 & 7.2 & 29.8 \\
\hline 4 & D06 & Abdominal pain, localized, other & 39 & 5.1 & 34.9 \\
\hline 5 & A91 & Abnormal result investigation NOS & 34 & 4.4 & 39.3 \\
\hline 5 & Nol & Headache & 34 & 4.4 & 43.8 \\
\hline 7 & DII & Diarrhea & 26 & 3.4 & 47.2 \\
\hline 8 & NI7 & Vertigo/dizziness & 23 & 3.0 & 50.2 \\
\hline 9 & $\mathrm{~A} 04$ & Weakness/tiredness general & 19 & 2.5 & 52.7 \\
\hline 10 & $\mathrm{R} 02$ & Shortness of breath/dyspnea & 16 & 2.1 & 54.8 \\
\hline II & D08 & Flatulence/gas/belching & 15 & 2.0 & 56.7 \\
\hline 12 & D09 & Nausea & 13 & 1.7 & 58.4 \\
\hline 12 & LOI & Neck symptom/complaint & 13 & 1.7 & 60.1 \\
\hline 12 & ROI & Pain, respiratory system & 13 & 1.7 & 61.8 \\
\hline 15 & L02 & Back symptom/complaint & 12 & 1.6 & 63.4 \\
\hline 15 & U98 & Abnormal urine test NOS & 12 & 1.6 & 65.0 \\
\hline 17 & DOI & Abdominal pain/cramps, general & 11 & 1.4 & 66.4 \\
\hline 17 & R2I & Throat symptom/complaint & 11 & 1.4 & 67.8 \\
\hline 19 & AlI & Chest pain NOS & 10 & 1.3 & 69.2 \\
\hline 19 & K07 & Swollen ankles/edema & 10 & 1.3 & 70.5 \\
\hline 21 & $\mathrm{~K} 02$ & Pressure/tightness of heart & 8 & 1.0 & 71.5 \\
\hline 21 & $\mathrm{R} 07$ & Sneezing/nasal congestion & 8 & 1.0 & 72.5 \\
\hline 21 & T8। & Goiter & 8 & 1.0 & 73.6 \\
\hline 24 & DI2 & Constipation & 7 & 0.9 & 74.5 \\
\hline 24 & L03 & Low back symptom/complaint & 7 & 0.9 & 75.4 \\
\hline 26 & B84 & Unexplained abnormal white cells & 6 & 0.8 & 76.2 \\
\hline 26 & D03 & Heartburn & 6 & 0.8 & 77.0 \\
\hline 26 & DIO & Vomiting & 6 & 0.8 & 77.8 \\
\hline 26 & D78 & $\begin{array}{l}\text { Neoplasm of digestive system, benign/ } \\
\text { unspecified }\end{array}$ & 6 & 0.8 & 78.6 \\
\hline 26 & K04 & Palpitations/awareness of heart & 6 & 0.8 & 79.3 \\
\hline 26 & K86 & Hypertension, uncomplicated & 6 & 0.8 & 80.1 \\
\hline \multirow[t]{2}{*}{26} & T03 & Loss of appetite & 6 & 0.8 & 80.9 \\
\hline & & Others & 146 & 19.1 & 100.0 \\
\hline Total & & & 765 & 100.0 & \\
\hline
\end{tabular}

Abbreviations: ICPC-2-R, International Classification of Primary Care, Revised Second edition; NOS, not otherwise specified; RFE, reason for encounter.

$\mathrm{n}=10$ ); back symptom/complaint (L02, n=8); and vertigo/ dizziness (N17, n=8).

Of the 765 patients, $210(27.5 \%)$ had visited another medical institution (in nearly all cases, clinics) for the same chief complaint before examination at our hospital. Figure 3 shows the number of patients who underwent examinations at another medical institution by RFE field. The highest proportion of patients being examined at another medical institution were respiratory ( $\mathrm{R} 51 / 146$ 34.9\%); endocrine, metabolic, and nutritional ( $\mathrm{T}$ 9/27 33.3\%); musculoskeletal (L 19/63 30.2\%); digestive (D 59/211 28.0\%); and general and unspecified (A 42/155 27.1\%). Antibiotics were prescribed at another medical institution to 53 patients in 5 fields. These antibiotics were clarithromycin, azithromycin, cefcapene, levofloxacin and so on (Figure 4). On observing the RFE, for respiratory ( $\mathrm{R} 27 / 5152.9 \%$ ) and general and unspecified (A 21/42 50.0\%), antibiotics were prescribed to $\geq 50 \%$ of patients. Of these, 34 of 88 patients (38.6\%) with an RFE of cough (R05) visited another medical institution, and 22 of those 34 patients $(64.7 \%)$ were prescribed antibiotics. The diagnoses of these 88 patients included upper respiratory infection, acute (common cold) (R74, $\mathrm{n}=35)$; asthma (R96, $\mathrm{n}=14)$; only classifiable as cough $(\mathrm{R} 05, \mathrm{n}=12)$; sinusitis acute/ chronic ( $\mathrm{R} 75, \mathrm{n}=5)$; pneumonia ( $\mathrm{R} 81, \mathrm{n}=4)$; esophagus disease (D84, $n=3)$; acute bronchitis/bronchiolitis $(R 78, n=3)$; and influenza $(\mathrm{R} 80, \mathrm{n}=3)$. Of the 85 patients who presented complaining of fever (A03), 25 (29.4\%) were examined at another medical institution. Of these 25 patients, $18(72.0 \%)$ 


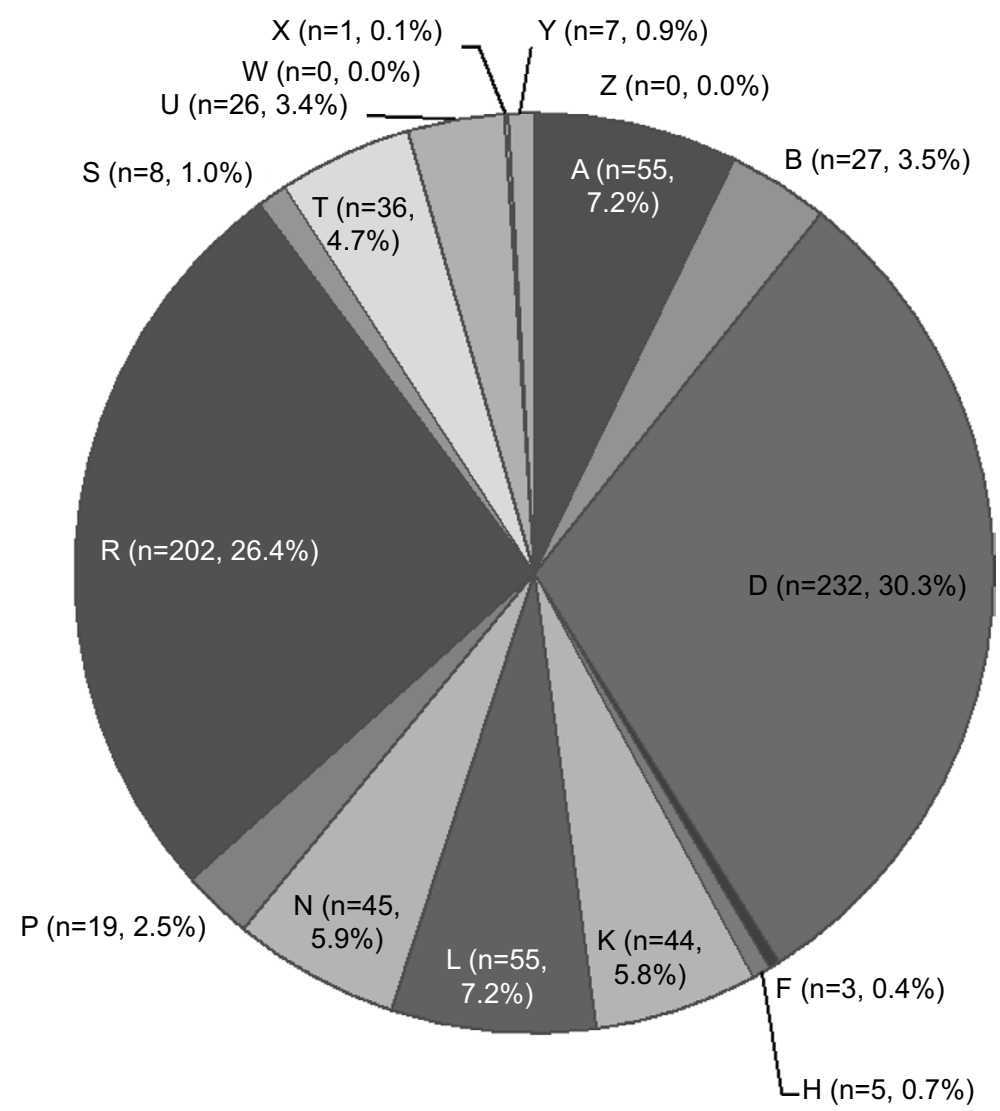

Figure 2 Frequency of diagnoses classified by the International Classification of Primary Care, Revised Second edition (ICPC-2-R) for 765 first-visit patients who presented to the Department of Internal Medicine at the Ikeda City Hospital without a referral on Fridays between April 2012 and March 2016.

Notes: $A=$ general and unspecified; $B=$ blood, blood-forming organs, and immune mechanisms; $D=$ digestive; $F=$ eye; $H=$ ear; $K=$ circulatory; $L=$ musculoskeletal; $N=$ neurological; $P=$ psychological; $R=$ respiratory; $S=$ skin; $T=$ endocrine, metabolic, and nutritional; $U=$ urological; $W=$ pregnancy, child-bearing, and family planning; $X=$ female genital; $Y=$ male genital; $Z$ = social problems.

were prescribed antibiotics. The diagnoses of the above 85 patients included upper respiratory infection, acute (R74, $\mathrm{n}=29$ ); influenza ( $R 80, \mathrm{n}=22)$; pneumonia ( $R 81, \mathrm{n}=6$ ); mild fever only classifiable as fever (A03, $n=4)$; pyelonephritis/ pyelitis (U70, $\mathrm{n}=4)$; infectious mononucleosis $(\mathrm{A} 75, \mathrm{n}=3)$; lymphadenitis, acute $(\mathrm{B} 70, \mathrm{n}=3)$; and gastroenteritis, presumed infection (D73, n=3).

There were 85 patients $(11.1 \%)$ who underwent an examination subsequent to a problem found during health checkup. Of these, 28 patients were classified as having "abnormal result investigation not otherwise specified (NOS)" (A91).

There were 479 patients $(62.4 \%)$ who underwent emergency testing at the initial examination and waited for the results. Emergency blood testing was performed for 318 patients $(41.6 \%)$, and emergency plain radiography was performed for 276 patients $(36.1 \%)$.

Table 3 shows the outcomes of the initial examination day. Those who were given appointments for a reexamination in an internal medicine department (i.e., the Department of Gastroenterology) at our acute care hospital and those for whom treatment ended on that same day accounted for $\sim 80 \%$ of all patients. Meanwhile, 33 patients (4.3\%) were hospitalized that day from "the Department of General Internal Medicine" Outpatient Clinic or had treatment in the Outpatient Emergency Department and were hospitalized that day at either our hospital or another medical institution. Including the 3 patients who were hospitalized at a later date, a total of 36 patients $(4.7 \%)$ were hospitalized.

There were 463 patients $(60.5 \%)$ who were prescribed medication on the initial examination day from the Department of General Internal Medicine or another department at our hospital and were examined on the same day.

There were 88 patients $(11.5 \%)$ in whom the examiner felt that the RFE was greatly influenced by a psychosomatic or psychiatric problem (e.g., depression, anxiety disorder, or somatization disorder). To investigate whether there were changes over time, the number of such cases was counted for the period from April 2012 to March 2013 and each following year, for a total of 4 years. The results indicated that such 
Table 2 Top 50 ICPC-2-R diagnosis codes of first-visit patients who presented to the Department of Internal Medicine at the Ikeda City Hospital without a referral on Fridays between April 2012 and March 2016

\begin{tabular}{|c|c|c|c|c|c|}
\hline Ranking & $\begin{array}{l}\text { ICPC-2-R } \\
\text { code }\end{array}$ & ICPC-2-R title & $\begin{array}{l}\text { Number of } \\
\text { cases }\end{array}$ & Percentage & $\begin{array}{l}\text { Cumulative } \\
\text { percentage }\end{array}$ \\
\hline $\mathrm{I}$ & R74 & Upper respiratory infection, acute & 86 & $1 \mathrm{I} .2$ & $1 \mathrm{I} .2$ \\
\hline 2 & D73 & Gastroenteritis, presumed infection & 49 & 6.4 & 17.6 \\
\hline 3 & D87 & Stomach function disorder & 33 & 4.3 & 22.0 \\
\hline 4 & $\mathrm{R} 80$ & Influenza & 28 & 3.7 & 25.6 \\
\hline 5 & D84 & Esophagus disease & 20 & 2.6 & 28.2 \\
\hline 6 & R96 & Asthma & 19 & 2.5 & 30.7 \\
\hline 7 & A97 & No disease & 15 & 2.0 & 32.7 \\
\hline 7 & D06 & Abdominal pain, localized, other & 15 & 2.0 & 34.6 \\
\hline 9 & $\mathrm{D} \mid 2$ & Constipation & 14 & 1.8 & 36.5 \\
\hline 9 & NOI & Headache & 14 & 1.8 & 38.3 \\
\hline II & D78 & Neoplasm of digestive system, benign/unspecified & 13 & 1.7 & 40.0 \\
\hline 12 & R05 & Cough & 12 & 1.6 & 41.6 \\
\hline 13 & R8I & Pneumonia & II & $\mathrm{I} .4$ & 43.0 \\
\hline 14 & B80 & Iron-deficiency anemia & 10 & 1.3 & 44.3 \\
\hline 14 & $\mathrm{D} 02$ & Abdominal pain, epigastric & 10 & 1.3 & 45.6 \\
\hline 16 & D97 & Liver disease NOS & 9 & 1.2 & 46.8 \\
\hline 16 & K86 & Hypertension, uncomplicated & 9 & 1.2 & 48.0 \\
\hline 16 & L85 & Acquired deformity of spine & 9 & 1.2 & 49.2 \\
\hline 16 & N95 & Tension headache & 9 & 1.2 & 50.3 \\
\hline 16 & T90 & Diabetes, non-insulin dependent & 9 & 1.2 & 51.5 \\
\hline 21 & L02 & Back symptom/complaint & 8 & 1.0 & 52.5 \\
\hline 21 & NI7 & Vertigo/dizziness & 8 & 1.0 & 53.6 \\
\hline 23 & All & Chest pain NOS & 7 & 0.9 & 54.5 \\
\hline 23 & D75 & Malignant neoplasm colon/rectum & 7 & 0.9 & 55.4 \\
\hline 23 & LOI & Neck symptom/complaint & 7 & 0.9 & 56.3 \\
\hline 23 & $\mathrm{~L} 03$ & Low back symptom/complaint & 7 & 0.9 & 57.3 \\
\hline 23 & ROI & Pain, respiratory system & 7 & 0.9 & 58.2 \\
\hline 23 & T8I & Goiter & 7 & 0.9 & 59.1 \\
\hline 29 & A91 & Abnormal result investigation NOS & 6 & 0.8 & 59.9 \\
\hline 29 & D98 & Cholecystitis/cholelithiasis & 6 & 0.8 & 60.7 \\
\hline 29 & K90 & Stroke/cerebrovascular accident & 6 & 0.8 & 61.4 \\
\hline 29 & R75 & Sinusitis acute/chronic & 6 & 0.8 & 62.2 \\
\hline 29 & R78 & Acute bronchitis/bronchiolitis & 6 & 0.8 & 63.0 \\
\hline 29 & U70 & Pyelonephritis/pyelitis & 6 & 0.8 & 63.8 \\
\hline 29 & U88 & Glomerulonephritis/nephrosis & 6 & 0.8 & 64.6 \\
\hline 36 & B70 & Lymphadenitis, acute & 5 & 0.7 & 65.2 \\
\hline 36 & D70 & Gastrointestinal infection & 5 & 0.7 & 65.9 \\
\hline 36 & H82 & Vertiginous syndromes & 5 & 0.7 & 66.5 \\
\hline 36 & K96 & Hemorrhoids & 5 & 0.7 & 67.2 \\
\hline 36 & LI4 & Leg/thigh symptom/complaint & 5 & 0.7 & 67.8 \\
\hline 36 & P74 & Anxiety disorder/anxiety state & 5 & 0.7 & 68.5 \\
\hline 36 & P76 & Depressive disorder & 5 & 0.7 & 69.2 \\
\hline 36 & T85 & Hyperthyroidism/thyrotoxicosis & 5 & 0.7 & 69.8 \\
\hline 44 & $\mathrm{~A} 03$ & Fever & 4 & 0.5 & 70.3 \\
\hline 44 & A75 & Infectious mononucleosis & 4 & 0.5 & 70.8 \\
\hline 44 & A76 & Viral exanthem, other & 4 & 0.5 & 71.4 \\
\hline 44 & A85 & Adverse effect medical agent & 4 & 0.5 & 71.9 \\
\hline 44 & B02 & Lymph gland(s) enlarged/painful & 4 & 0.5 & 72.4 \\
\hline 44 & DOI & Abdominal pain/cramps, general & 4 & 0.5 & 72.9 \\
\hline 44 & D08 & Flatulence/gas/belching & 4 & 0.5 & 73.5 \\
\hline 44 & DII & Diarrhea & 4 & 0.5 & 74.0 \\
\hline 44 & D86 & Peptic ulcer, other & 4 & 0.5 & 74.5 \\
\hline 44 & D92 & Diverticular disease & 4 & 0.5 & 75.0 \\
\hline 44 & L99 & Musculoskeletal disease, other & 4 & 0.5 & 75.6 \\
\hline 44 & N06 & Sensation disturbances, other & 4 & 0.5 & 76.1 \\
\hline 44 & P75 & Somatization disorder & 4 & 0.5 & 76.6 \\
\hline 44 & $\mathrm{R} 72$ & Strep throat & 4 & 0.5 & 77.1 \\
\hline 44 & R99 & Respiratory disease, other & 4 & 0.5 & 77.6 \\
\hline \multirow[t]{2}{*}{44} & U99 & Urinary disease, other & 4 & 0.5 & 78.2 \\
\hline & & Others & 167 & 21.8 & 100.0 \\
\hline Total & & & 765 & 100.0 & \\
\hline
\end{tabular}

Abbreviations: ICPC-2-R, International Classification of Primary Care, Revised Second edition; NOS, not otherwise specified. 


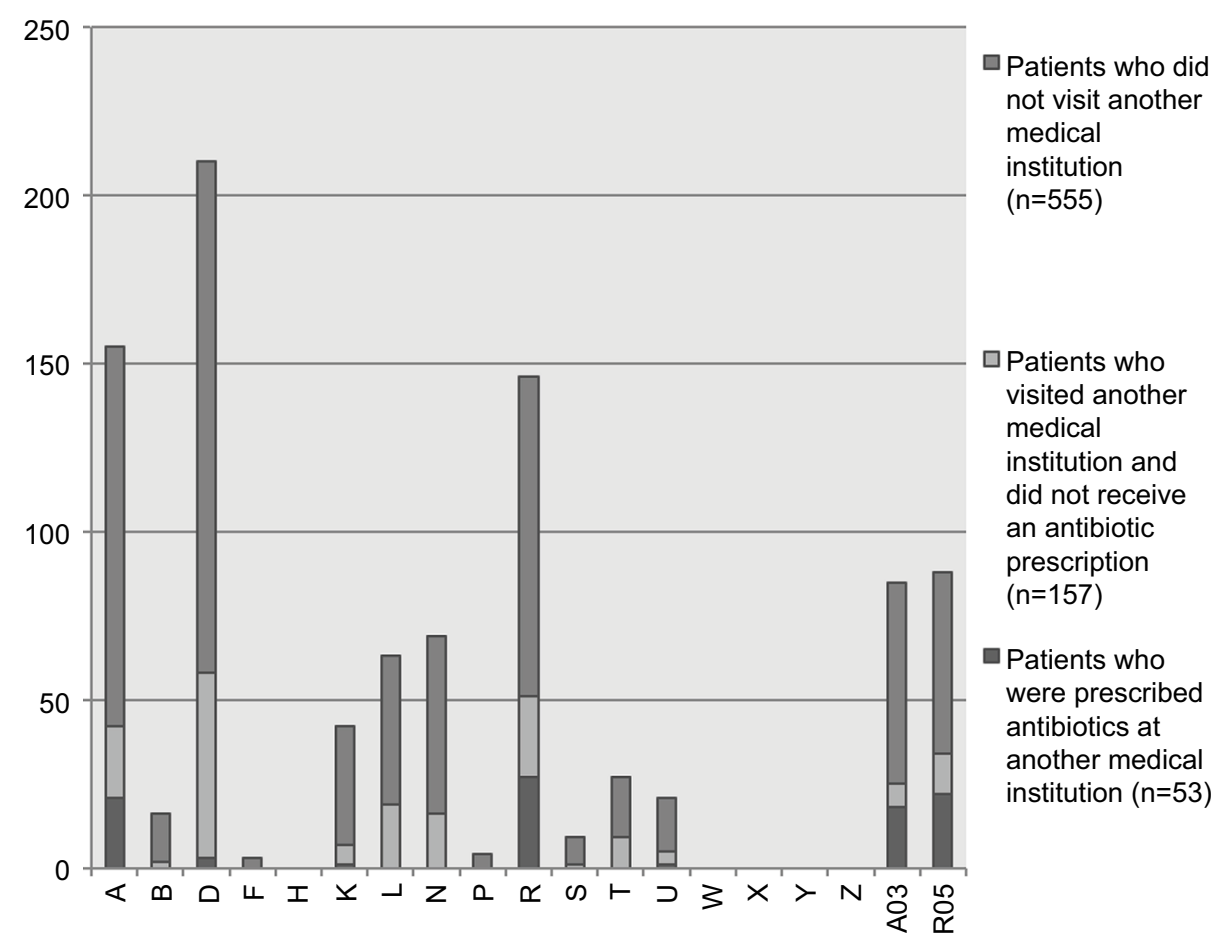

Figure 3 First-visit patients $(n=765)$ who presented to the Department of Internal Medicine at the Ikeda City Hospital without a referral on Fridays between April 2012 and March 2016, who visited another medical institution, and who were or were not prescribed antibiotics.

Notes: Results are categorized by reason for encounter fields classified by the International Classification of Primary Care, Revised Second edition (ICPC-2-R) and whether or not patients who visited another medical institution before presenting to our hospital were prescribed antibiotics by that institution. RFEs on the far right edge exhibit an $A 03$ and R05 overlap with the number of cases of $A$ and $R$ on the left. Legend ( $n=$ the number of patients who did not visit another medical institution, the number of patients who visited another medical institution and did not receive an antibiotics prescription, and the number of patients who were prescribed antibiotics at another medical institution, respectively.): $A=$ general and unspecified $(n=113,21,21) ; B=$ blood, blood-forming organs, and immune mechanisms $(n=14,2,0) ; D=$ digestive $(n=152$, $55,3) ; F=$ eye $(n=3,0,0) ; H=$ ear $(n=0,0,0) ; K=$ circulatory $(n=35,6, I) ; L=$ musculoskeletal $(n=44,19,0) ; N=$ neurological $(n=53,16,0) ; P=p s y c h o l o g i c a l ~(n=4,0,0) ;$ $R=$ respiratory $(n=95,24,27) ; S=$ skin $(n=8, I, 0) ; T=$ endocrine, metabolic, and nutritional $(n=18,9,0) ; U=$ urological $(n=16,4, I) ; W=$ pregnancy, child-bearing, and family planning $(n=0,0,0) ; X=$ female genital $(n=0,0,0) ; Y=$ male genital $(n=0,0,0) ; Z=$ social problems $(n=0,0,0) ; A 03=$ fever $(n=60,7,18) ; R 05=$ cough $(n=54,12,22)$. Abbreviation: RFE, reason for encounter.

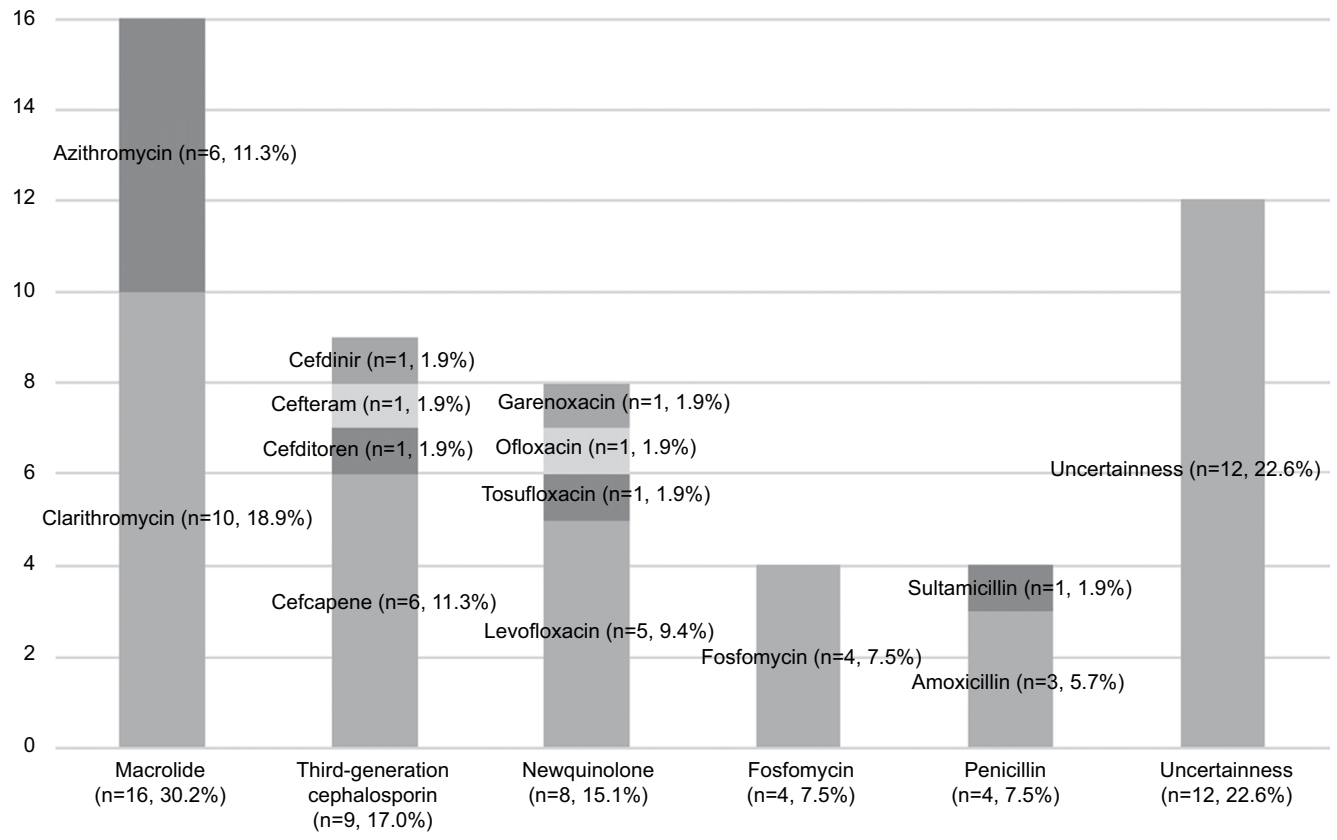

Figure 4 Antibiotics prescribed at another medical institution to 765 first-visit patients who presented to the Department of Internal Medicine at the Ikeda City Hospital without a referral on Fridays between April 2012 and March $2016(n=53)$. 
Table 3 Outcomes of first-visit patients who presented to the Department of Internal Medicine at the lkeda City Hospital without a referral on Fridays between April 2012 and March 2016

\begin{tabular}{lll}
\hline Outcome & Number of cases & Percentage \\
\hline End of treatment & $30 I$ & 39.3 \\
Appointment for internal medicine treatment & 310 & 40.5 \\
Referral to another medical institution & 64 & 8.4 \\
Appointment for internal medicine treatment + referral to another medical institution & 19 & 2.5 \\
Admission to our hospital on that day & 15 & 2.0 \\
Planned admission to our hospital & 3 & 0.4 \\
Emergency outpatient $\rightarrow$ return home & 7 & 0.9 \\
Emergency outpatient $\rightarrow$ admission to our hospital on that day & 15 & 2.0 \\
Emergency outpatient $\rightarrow$ transfer for admission at another hospital & 3 & 0.4 \\
Treatment at another department within the hospital & 23 & 3.0 \\
Treatment at another department within the hospital + appointment for internal medicine treatment & 4 & 0.5 \\
Treatment at another department within the hospital + referral to another medical institution & 1 & 0.1 \\
Total & 765 & 100.0 \\
\hline
\end{tabular}

patients annually accounted for $28 / 223$ cases (12.6\%), 27/173 (15.6\%), 18/163 (11.1\%), and 15/206 (7.3\%), respectively.

\section{Discussion}

The objective of this study was to examine the medical demands placed on medium-sized acute care hospitals in Japan. We used the ICPC-2-R to analyze RFEs and diagnoses. The ICPC-2-R is a useful tool for understanding medical needs. ${ }^{5}$ Previously, there have been reports in Japan regarding RFEs of outpatients at clinics and hospitals; $;, 7$ however, there are few reports in the English literature on mediumsized hospitals. In the present study, we primarily focused on patients who presented without a referral and analyzed RFEs and diagnoses on the basis of the ICPC-2-R. Takeshima et al used the International Classification of Primary Care, Second Edition (ICPC-2) ) $^{8}$ to analyze RFEs and diagnoses of outpatients at a small-sized 90-bed hospital in Japan. ${ }^{9}$ Their results showed that the respiratory $(\mathrm{R})$, general and unspecified (A), and digestive (D) categories were common RFEs. These results are consistent with those of the present study, thereby revealing the commonality of RFEs.

With regard to the diagnoses, the most common result was digestive (D), followed by respiratory (R). It is important to note that general and unspecified (A) was less than other RFE results. This may have been greatly influenced by the fact that many patients with an RFE of fever (A03), which was the most common type of general and unspecified (A) RFE, were subsequently diagnosed with upper respiratory infection, acute (R74) or influenza (R80), and the fact that patients with an RFE of abnormal result investigation NOS (A91) included many patients diagnosed with a disease of the digestive (D) system (e.g., liver disease NOS [D97]). These results were also consistent with those reported by Takeshima et al. ${ }^{9}$ On the other hand, the frequency of dermatological and orthopedic problems was relatively higher at outpatient clinics than at hospitals. ${ }^{6,7}$ It indicates that patients expect solutions about fields of health problems from their primary care clinics rather than internal medicine departments of hospitals. However, the Ikeda City Hospital has the Department of Dermatology and the Department of Orthopedics. There are no major change on correspondence about fields of health problems between our hospital and primary care clinics.

Another objective of this study was to highlight the problem of overprescription of antibiotics in the primary care setting in Japan. We investigated whether patients had visited another medical institution for the same RFE and had been prescribed antibiotics before undergoing an examination at our hospital. However, we did not investigate why the patients had decided to present to our hospital based on their own judgment, despite not receiving a referral. It is possible that the patients were dissatisfied with the treatment that they had received for various reasons (e.g., their symptoms were not improving). Visiting both a clinic and a hospital without a referral is medical-economically not efficient and is wasteful on time. Redefining the gatekeeper function of primary care physicians may improve this issue. Figure 4 shows the results of our investigation regarding whether the proportions of patients who underwent an examination at another medical institution varied according to disease field. However, we did not detect any fields with a particularly high proportion of patients having undergone an examination at another medical institution. While patients with RFEs of respiratory $(\mathrm{R})$ and general and unspecified (A) - in particular, cough (R05) and fever (A03) were frequently prescribed antibiotics when examined at other medical institutions; only a few patients with an RFE of cough (R05) or fever (A03) were subsequently diagnosed with a 
bacterial infection at our hospital. Of those, patients diagnosed with lymphadenitis, acute (B70), sinusitis acute/chronic (R75), acute bronchitis/bronchiolitis (R78), pneumonia (R81), or pyelonephritis/pyelitis (U70), 12 (13.6\%) of 88 patients with cough (R05) and 13 (15.3\%) of 85 patients with fever (A03) needed antibiotics. Although the relationship with treatment satisfaction remains unclear, this appears to be one problematic point associated with primary care in this region. However, if the antibiotics had been effective, the need to visit our hospital should have decreased. On examination of data from Japanese medical wholesale dealers in 2013, it has been reported that, in Japan, $\sim 2$ million people are prescribed antibiotics each day. ${ }^{10}$ Higashi et al reported from a cross-sectional analysis of insurance claims to an employer-sponsored health insurance plan in Japan that antibiotics were prescribed in $60 \%$ of 2577 claims for nonbacterial upper respiratory tract infections. ${ }^{11}$ Their data were collected in 2005. In the Choosing Wisely initiative conducted in the USA, the Infectious Diseases Society of America is advising "avoiding prescribing antibiotics for upper respiratory infections." The fact that more judicious use of antibiotics is being promoted suggests that the unnecessary prescription of antibiotics for acute upper respiratory tract infections is also occurring frequently in the USA. ${ }^{12}$ In 2016, the Japanese government announced an action plan that included reducing unnecessary prescriptions of antibiotics. ${ }^{13}$ Thus, reducing unnecessary antibiotics prescriptions is an international issue in primary care. On the contrary, a cohort study of 610 UK general practices from the UK Clinical Practice Research Datalink shows that if a general practice with an average list size of 7000 patients reduces the proportion of respiratory tract infection consultations with antibiotics prescribed by $10 \%$, then it might observe 1.1 cases of pneumonia each year. ${ }^{14}$ Caution might be required in subgroups at higher risk for pneumonia.

In Japan, the government recommends that people with no particular subjective symptoms undergo regular health checkups. ${ }^{1}$ Consequently, many individuals with no particular subjective symptoms who undergo regular checkups also undergo detailed testing for unsymptomatic hypertension or undergo detailed testing due to an abnormal result. In the present study, $11.1 \%$ of the patients presented as a result of an abnormality found on a health checkup. In an effort to improve quality and reduce costs, it is important for doctors to offer appropriate treatments according to evidence-based medicine. Takeshima et al also reported that a common RFE was abnormal result investigation NOS (A91). ${ }^{9}$ Thus, it is problematic that abnormal result investigation NOS (A91) is not included in the "Goals of Clinical Clerkship" established by the Ministry of Health, Labour and Welfare for clinical internists to experience in the first 2 years after graduating from medical school and in the "Model Core Curriculum in Medical Education, the guideline for the educational programme," in which the Ministry of Education, Culture, Sports, Science and Technology describes the minimum educational content that medical students should learn by the time that they graduate. ${ }^{15,16}$ Moreover, we want to point out that primary care clinics can often correspond to the RFE: abnormal result investigation NOS (A91).

Emergency testing was performed on the majority of patients $(62.4 \%)$. While the results were affected by the treatment attitude, which tends to depend on testing by the individual examiner, they may also reflect the fact that patients who undergo an examination at a general hospital rather than a medical clinic tend to desire detailed testing.

Although it is difficult to compare our results with those of other studies because of the small number of relevant reports, one noticeable trend was the high percentage of patients for whom hospitalization was determined on the day of initial examination (4.7\%). Wooldridge et al reported an analysis of diagnoses and treatment times in 263 cases over 8 days at a rural medical clinic in Japan and noted that no patients were hospitalized..$^{17}$ It is possible that some patients decided to undergo an examination at an acute care hospital because of severe symptoms.

It was found that $60.5 \%$ of patients were prescribed medication on the initial examination day; however, it is also difficult to compare these results as there are few other available reports.

In $11.5 \%$ of patients, it was thought that the RFE may have been greatly influenced by a psychosomatic condition. One limitation of our study was that the results were based on the subjective judgment of a nonspecialist without specific scores or criteria. However, a certain level of reproducibility was noted, with no great variation in the proportion of such patients throughout the observation period. If we had used the Somatic Symptom Scale-8 or Somatic Symptoms Experiences Questionnaire, we could have more accurately diagnosed psychosomatic conditions. ${ }^{18,19}$ Ishikawa et al reported that $12.4 \%$ of first-visit patients to a general medicine outpatient clinic at a university hospital in Japan had psychiatric disorders. ${ }^{20}$ Although described as "psychiatric disorders" in Ishikawa's report, they included, beginning with the most common, depression, anxiety disorder, and somatization disorder, and these could also be considered psychosomatic disorders. Therefore, the results are consistent with those of the present study.

This study has some other limitations. Only 1 doctor's examinations were included. Therefore, it is likely that the 
diagnoses and clinical decisions were affected by the examiner's clinical bias. However, because RFEs were based on the patients' complaints, this bias was likely weak. Because the examination day was limited to Fridays, the results may have been affected by patient treatment-seeking behavior (e.g., the frequency of visiting another medical institution), although this was unlikely to affect RFE or diagnosis. Moreover, most of clinics near our hospital were closed on Sundays, and on Wednesday, Thursday, and Saturday afternoons. Because the period for accumulating results was precisely 4 years, it is unlikely that results were affected by seasonal variation or the epidemic status of infections. This was a single-institution study, and it is uncertain whether the data were typical of other hospitals in Japan. However, as we discussed above, our results were similar to other Japanese reports. ${ }^{6,7,9,11,17,20}$

In the future, we want to examine the reasons why some patients visited our hospital without a referral after having visited another medical institution. We would like to examine changes in RFEs and diagnoses with regard to aging as well. Additionally, through discussions with doctors in this region, we hope to encourage the adequate use of antibiotics and verify progress in that regard.

\section{Conclusion}

We used the ICPC-2-R to analyze first-visit patients who presented without a referral to the Department of Internal Medicine at a medium-sized acute care hospital in Japan. Common RFEs consisted of abdominal pain (D02, D06, and D01), cough (R05), and fever (A03). Common diagnoses included upper respiratory infection, acute (R74); gastroenteritis, presumed infection (D73); stomach function disorder (D87); and influenza (R80).

The results suggest a tendency toward the excessive prescription of antibiotics for some conditions (e.g., cough [R05] and fever [A03]) by primary care physicians. There is no clear boundary to separate the roles of clinics versus hospitals. However, there is a possibility that patients with more serious illness chose our hospital rather than clinics by themselves.

\section{Acknowledgments}

We would like to extend our deepest gratitude toward Dr Masaki Amenomori, who gave the authors guidance regarding general medical treatment, and nurses Mieko Maeyama and Eriko Okiie, who assisted with general outpatient treatment.

We also thank Crimson Interactive Pvt. Ltd. (Ulatus) www.ulatus.jp - and Forte Science Communications - www. forte-science.co.jp - for their assistance in manuscript translation and editing.

\section{Author contributions}

NK treated all patients, accumulated the data, and wrote the draft. KH, MM, SM, and TU participated in discussions regarding treatment, accumulation of data, and the Discussion section. MM accumulated the data. All authors contributed toward data analysis, drafting and revising the paper and agree to be accountable for all aspects of the work.

\section{Disclosure}

The authors report no conflicts of interest in this work.

\section{References}

1. Ikeda N, Saito E, Kondo N, et al. What has made the population of Japan healthy? Lancet. 2011;378(9796):1094-1105.

2. Hashimoto H, Ikegami N, Shibuya K et al. Cost containment and quality of care in Japan: is there a trade-off? Lancet. 2011;378(9797): 1174-1182.

3. Mark Britnell. Fragmentation. In: Mark Britnell. In Search of The Perfect Health System. London: Palgrave Macmillan; 2015:16-17.

4. Ministry of Health, Labour and Welfare [homepage on the Internet]. Tokyo, Japan: Fee for Treatment of Patients' Choice. Available from: http://www.mhlw.go.jp/file/06-Seisakujouhou-10800000-Iseikyoku/0000056829.pdf. Accessed November 13, 2016.

5. WONCA International Classification Committee (WICC): ICPC-2-R: International Classification of Primary Care. Revised Second Edition. Oxford: Oxford Medical Publications; 2005.

6. Yamada T, Yoshimura M, Nago N et al. [What are common diseases and common health problems? The use of ICPC in the community-based project]. Jpn J Prim Care. 2000;23(1):80-89. Japanese.

7. Tanaka K, Nomaguchi S, Matsumura S, Fukuhara S. [Ranking the frequency of patient illness at primary care clinics]. Jpn J Prim Care. 2007;30(4):344-351. Japanese.

8. WONCA International Classification Committee (WICC): ICPC-2: International Classification of Primary Care. Second Edition. Oxford: Oxford Medical Publications; 1998.

9. Takeshima T, Kumada M, Mise J, et al. Reasons for encounter and diagnoses of new outpatients at a small community hospital in Japan: an observational study. Int J Gen Med. 2014;7:259-269.

10. MurakiY, Tanabe M, Watanabe T, et al. [The trend of all antibiotics use in Japan (2009-2013)]. Jpn JEnviron Infect. 2016;31(Suppl):268. Japanese.

11. Higashi T, Fukuhara S. Antibiotic prescriptions for upper respiratory tract infection in Japan. Intern Med. 2009;48(16):1369-1375.

12. Infectious Diseases Society of America [homepage on the Internet]. Arlington, VA: Choosing Wisely: Infectious Diseases Society of America. Available from: http://www.choosingwisely.org/societies/ infectious-diseases-society-of-america/. Accessed May 14, 2016.

13. Ministerial Meeting on Measures on Emerging Infectious Diseases [homepage on the Internet]. Tokyo, Japan: [National Action Plan on Antimicrobial Resistance (2016-2020).] Available from: http:// www.kantei.go.jp/jp/singi/kokusai_kansen/pdf/yakuzai_honbun.pdf. Accessed July 24, 2016. Japanese.

14. Gulliford MC, Moore MV, Little P, et al. Safety of reduced antibiotic prescribing for self limiting respiratory tract infections in primary care: cohort study using electronic health records. BMJ. 2016;354:i3410.

15. Ministry of Health, Labour and Welfare [homepage on the Internet]. Tokyo, Japan: [Goals of Clinical Clerkship] Available from: http:// www.mhlw.go.jp/file/05-Shingikai-10801000-Iseikyoku-Soumuka/0000102821.pdf. Accessed May 14, 2016. Japanese. 
16. Ministry of Education, Culture, Sports, Science and Technology [homepage on the Internet]. Tokyo, Japan: [Model Core Curriculum in Medical Education, the guideline for the educational programme] Available from: http://www.mext.go.jp/b_menu/shingi/chousa/koutou/033-1/ toushin/1304433.htm. Accessed May 14, 2016. Japanese.

17. Wooldridge AN, Arató N, Sen A, Amenomori M, Fetters MD. Truth or fallacy? Three hour wait for three minutes with the doctor: findings from a private clinic in rural Japan. Asia Pac Fam Med. 2010;9(1):11.

18. Gierk B, Kohlmann S, Kroenke K, et al. The somatic symptom scale-8 (SSS-8): a brief measure of somatic symptom burden. JAMA Intern Med. 2014;174(3):399-407.
19. Herzog A, Voigt K, Meyer B, et al. Psychological and interactional characteristics of patients with somatoform disorders: validation of the Somatic Symptoms Experiences Questionnaire (SSEQ) in a clinical psychosomatic population. J Psychosom Res. 2015;78(6):553-562.

20. Ishikawa Y, Takeshima T, Mise J, Ishikawa S, Matsumura M. Physical symptoms in outpatients with psychiatric disorders consulting the general internal medicine division at a Japanese university hospital. Int J Gen Med. 2015;8:261-266.
International Journal of General Medicine

\section{Publish your work in this journal}

The International Journal of General Medicine is an international, peer-reviewed open-access journal that focuses on general and internal medicine, pathogenesis, epidemiology, diagnosis, monitoring and treatment protocols. The journal is characterized by the rapid reporting of reviews, original research and clinical studies across all disease areas.

\section{Dovepress}

The manuscript management system is completely online and includes a very quick and fair peer-review system, which is all easy to use. Visit http://www.dovepress.com/testimonials.php to read real quotes from published authors.

Submit your manuscript here: https://www.dovepress.com/international-journal-of-general-medicine-journal 